\title{
Limitations and possibilities of low cell number ChIP-seq
}

\author{
Gregor D Gilfillan ${ }^{1 *}$, Timothy Hughes', Ying Sheng ${ }^{1}$, Hanne S Hjorthaug ${ }^{1}$, Tobias Straub², Kristina Gervin ${ }^{3}$, \\ Jennifer R Harris ${ }^{4}$, Dag E Undlien ${ }^{3}$ and Robert Lyle ${ }^{1 *}$
}

\begin{abstract}
Background: Chromatin immunoprecipitation coupled with high-throughput DNA sequencing (ChIP-seq) offers high resolution, genome-wide analysis of DNA-protein interactions. However, current standard methods require abundant starting material in the range of 1-20 million cells per immunoprecipitation, and remain a bottleneck to the acquisition of biologically relevant epigenetic data. Using a ChIP-seq protocol optimised for low cell numbers (down to 100,000 cells / IP), we examined the performance of the ChIP-seq technique on a series of decreasing cell numbers.
\end{abstract}

Results: We present an enhanced native ChIP-seq method tailored to low cell numbers that represents a 200-fold reduction in input requirements over existing protocols. The protocol was tested over a range of starting cell numbers covering three orders of magnitude, enabling determination of the lower limit of the technique. At low input cell numbers, increased levels of unmapped and duplicate reads reduce the number of unique reads generated, and can drive up sequencing costs and affect sensitivity if ChIP is attempted from too few cells.

Conclusions: The optimised method presented here considerably reduces the input requirements for performing native ChIP-seq. It extends the applicability of the technique to isolated primary cells and rare cell populations (e.g. biobank samples, stem cells), and in many cases will alleviate the need for cell culture and any associated alteration of epigenetic marks. However, this study highlights a challenge inherent to ChIP-seq from low cell numbers: as cell input numbers fall, levels of unmapped sequence reads and PCR-generated duplicate reads rise. We discuss a number of solutions to overcome the effects of reducing cell number that may aid further improvements to ChIP performance.

Keywords: PCR duplicates, Redundant reads, HTS, NGS, Next generation sequencing, Micro-ChIP, N-ChIP, Native ChIP, Location analysis, Histone

\section{Background}

Chromatin immunoprecipitation (ChIP) is used to determine the genomic interaction sites between nuclear proteins and nucleic acids. Standard ChIP protocols used for genome-wide studies typically require large quantities of starting material, in the range of $10^{7}$ cells. The amount of material immunoprecipitated varies depending on the target protein and antibody employed, but is usually in the range of a few hundred picograms to tens of nanograms.

\footnotetext{
* Correspondence: gregor.gilfillan@medisin.uio.no; robert.lyle@medisin.uio.no 'Department of Medical Genetics, Oslo University Hospital, Oslo, Norway Full list of author information is available at the end of the article
}

Over the past decade, genome-wide analysis of ChIP material has been possible by employing whole genome amplification techniques to produce microgram quantities of DNA for hybridisation to microarrays, commonly referred to as ChIP-chip. Recently, the application of high-throughput sequencing (HTS) to analyse the immunoprecipitates, commonly referred to as ChIP-seq, has replaced ChIP-chip as the preferred means of harvesting genome-wide data, and confers a number of advantages including higher resolution, improved quantification range, greater genome coverage, fewer artifacts, and lower cost [1]. However, the library preparation methods required to render immunoprecipitated DNA ready for HTS sequencing involve inefficient enzymatic steps and multiple purifications, each

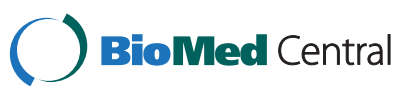


resulting in sample loss. As a result, ChIP-seq requires a similar starting amount of immunoprecipitated DNA (1-10 ng) to ChIP-chip, and nonetheless involves many cycles of PCR (typically 15-18 cycles). These limitations mean that it is still challenging to apply ChIP-seq to low numbers of cells.

There have been several recent papers detailing ChIP protocols applicable to low cell numbers (down to 100 cells) based on the inclusion of carrier DNA [2] or improvements in the efficiency of the immunoprecipitations themselves [3-7]. However, these techniques have been limited to locus-specific analysis of the precipitates by quantitative PCR. By incorporating whole genome amplification (WGA) methods, these techniques have been extended to allow genome-wide analysis by ChIPchip from as few as 10,000 cells [8] or 1,000 cells [9].

Three recent publications detail the development of ChIP-seq protocols for use with low cell numbers, all based on formaldehyde crosslinked chromatin: Using standard Illumina library preparation procedures, Hitchler \& Rice demonstrated ChIP-seq from $1 \times 10^{6}$ human stem cells and 5 ng immunoprecipitated DNA [10]. Greater reductions have been possible using alternative library preparation methods, where cell requirements have been lowered to 10,000 [11] (the method is also presented in more detail elsewhere [12]), and even 5000 [13], with input DNA amounts below 50 pg. However, these methods employ either lengthy linear amplification procedures, or primer extension ( 4 cycles) and 15 cycles of PCR - all prior to a standard Illumina library prep entailing a further 17-18 cycles of PCR.

An alternative method of performing ChIP avoids the use of formaldehyde cross-linking and is thus known as native ChIP (N-ChIP) [14-17]. The advantages of $\mathrm{N}$ ChIP over cross-linked ChIP (X-ChIP) include higher resolution, and lack of unspecific interaction caused by formaldehyde crosslinking. It has also been suggested that the N-ChIP method is more sensitive than X-ChIP, as epitopes may not be masked by cross-linked proteins or be themselves denatured by formaldehyde [15] making N-ChIP ideally suited to studies aiming to examine small cell numbers. However, N-ChIP is generally considered only applicable to histone proteins, although successful N-ChIP of MeCP2 [18] and a handful of transcription factors including Runx2, Dlx6 and Sin3a have been reported [19].

We present here an N-ChIP method for genome-wide analysis by ChIP-seq, optimised for use with low starting cell numbers (here 200,000, divided into two immunoprecipitations of 100,000 each). This demonstrates ChIP-seq with 200-times fewer cells than a previously published method used as a benchmark for comparison. The performance of the optimised method was evaluated for read mapping, sensitivity and specificity at a range of starting cell numbers covering three orders of magnitude, starting with the published amount of $2 \times 10^{7}$ cells / IP and reduced to a point where sensitivity was compromised, to determine the limits of the technique.

\section{Results}

The ChIP method described here was developed using the N-ChIP method of Zhao and colleagues [20,21] as a starting point. We therefore set the existing technique, using the published amount of $2 \times 10^{7}$ cells per immunoprecipitation, as a benchmark against which to compare the performance of our method at a series of decreasing cell numbers. The new method presented here significantly shortens the procedure by eliminating the need for dialysis, and incorporates modifications optimised for low cell numbers.

Chromatin prepared using the two methods was prepared from cultured $\mathrm{CD}^{+}$lymphocytes, and immunoprecipitated with anti-H3K4me3 antibody. Enrichment at positive and negative control loci (see methods section) was measured by quantitative PCR prior to generation of Illumina sequencing libraries. Each ChIP-seq library was then sequenced on a single lane of an Illumina GAIIx sequencer, and generated chromatin profiles typical of H3K4 trimethylation, from which peaks were called. At this point it was determined that additional sequencing was required to saturate peak calling (see below) in the lowest cell number employed, so additional sequencing was performed for this sample using a single lane of an Illumina HiSeq 2000 machine.

The total number of reads generated for each library and the results of aligning these libraries to the human genome are summarized in Figure 1. As cell numbers are reduced, the number of unmapped reads increases. In addition, the percentage of mapped reads derived from duplicate reads increases. A sample of unmapped reads was aligned against the GenBank nucleotide database [22] using blast [23]. The results showed that a small proportion of unmapped reads in all cases represent those containing sequencing errors that fail to map to the human genome (the BWA algorithm does not tolerate more than 2 mismatches in its $32 \mathrm{bp}$ seed). The remainder of unmapped reads, which increase with decreasing input cell number, fail to map with high confidence to any sequence in the GenBank database and are apparently PCR amplification artifacts. The increased level of duplicate reads seen at lower cell numbers is also assumed to be introduced during the PCR amplification (18 cycles) required as part of Illumina library preparation. The same number of PCR cycles were applied to each sample, to facilitate inter-sample comparisons. A disadvantage of this approach is that a greater number of cycles than necessary to generate sufficient library to sequence, were applied to the higher cell number 


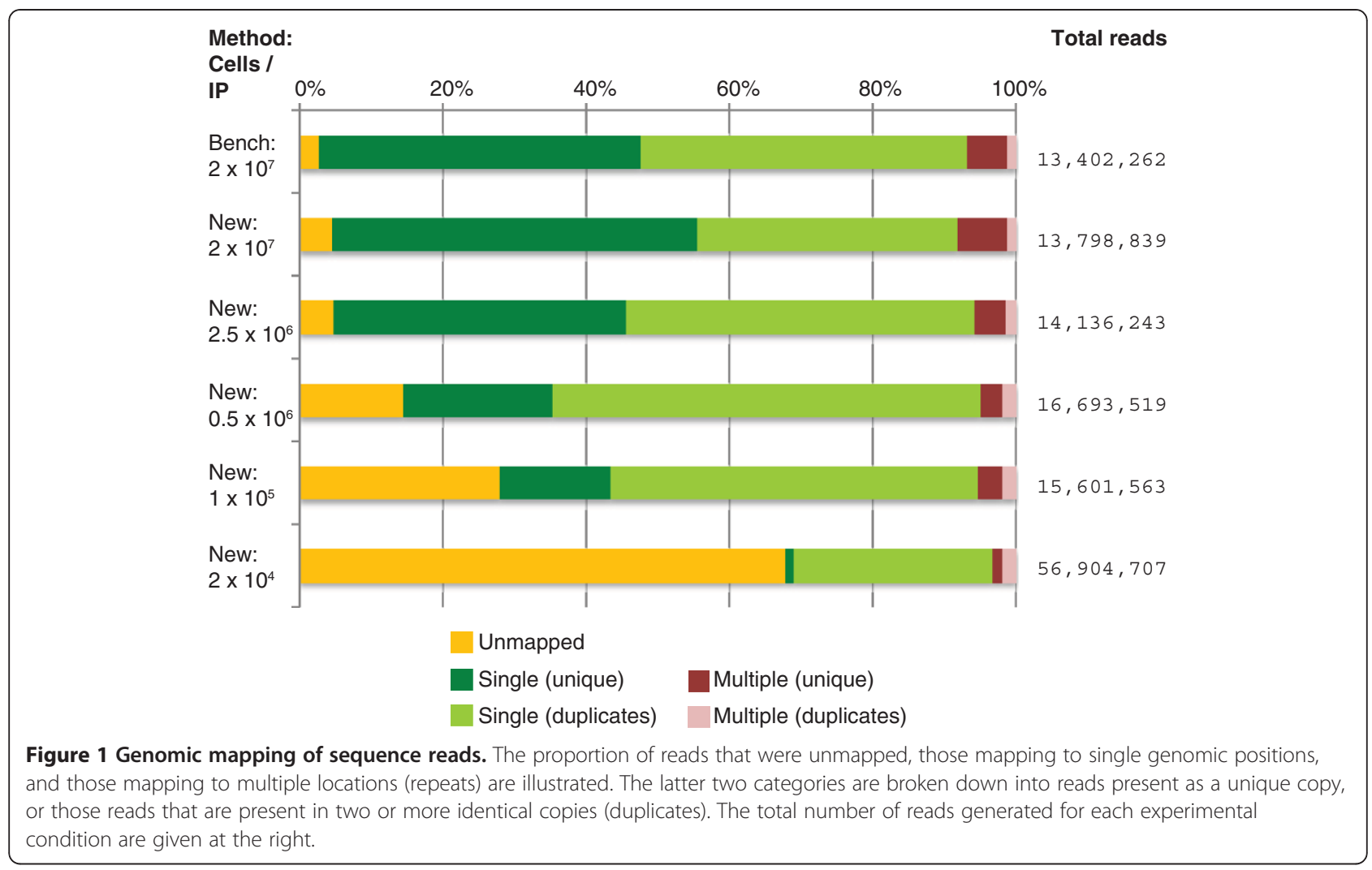

samples. Despite this, only in the low cell number samples did the decreased amount and complexity of the input material, lead to high proportions of duplication during amplification.

ChIP-seq profiles from each experiment can be seen in Figure $2 \mathrm{a}$ and $2 \mathrm{~b}$, and by visual inspection, peaks of H3K4me3 can be seen to occupy promoter regions as expected from previous studies [24]. To demonstrate the increased sensitivity of the new method relative to the benchmark, an additional profile generated using the benchmark at a low cell number is shown (Figure 2a and 2b). The resolution afforded by the MNase digestion allows identification of individual nucleosome positions (Figure 2b). The relationship between H3K4me3 and transcription start sites (TSS) was confirmed by plotting H3K4me3 levels relative to transcription start sites (Figure 2c), which reproduces earlier findings showing depletion of histones at the TSS itself, and a series of clearly positioned nucleosomes upstream and downstream [20]. Furthermore, immunoprecipitation with H3K4me3 is strongest at highly expressed genes, and diminishes with decreasing expression level (Figure 2c).

To define genomic regions of H3K4 trimethylation, peak calling was performed using MACS [26], using only uniquely mapping, non-duplicate reads. Performing peak calling whilst including duplicate reads led to the appearance of high numbers of non-specific peaks, particularly in the lowest cell number sample (data not shown). Calling peaks when correcting for background control (sequencing libraries prepared from each sample's ChIP input DNA) made negligible difference to the number of peaks recognised; $0.03-1.43 \%$ of peaks were no longer called when using a control dataset. The total number of peaks called for each cell number is summarized in Table 1. The number of peaks called falls appreciably only at the lowest cell input number tested, to below $75 \%$ of the number called in the benchmark. Crucially, despite the lower numbers of uniquely mapped reads recovered from the lowest cell number sample, peak calling was saturated for all samples: When decreasing proportions of total available reads were used to call peaks (Figure 3a), the absence of a reciprocal relationship between read count and peaks called indicates that all samples are approaching saturation (i.e. where adding more reads will not increase the number of peaks called). Therefore, the lower number of peaks called using only 20,000 cells / IP is due to the reduced number of useful reads (non duplicated and uniquely mapping) available as cell numbers fall, and not simply because more sequencing is required.

Using the method of Zhao and colleagues as a benchmark, the overlap with peaks called from the new method was evaluated as a measure of sensitivity (Table 1). Sensitivity was well maintained down to 
(a) Benchmark $2 \times 10^{7}$

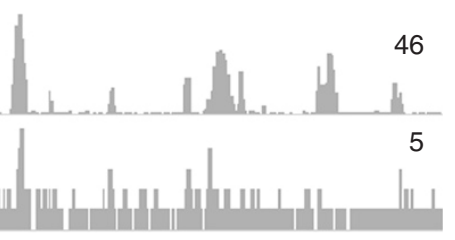

New $2 \times 10^{7}$

Benchmark $0.5 \times 10^{6}$

$\mid$

New $2.5 \times 10^{6}$

New $0.5 \times 10^{6}$

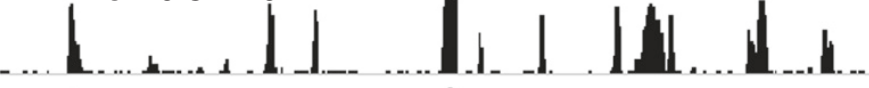

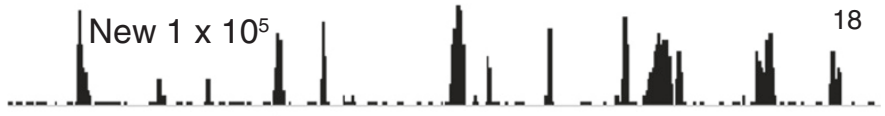

New $2 \times 10^{4} \mid$

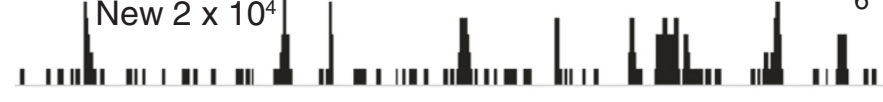

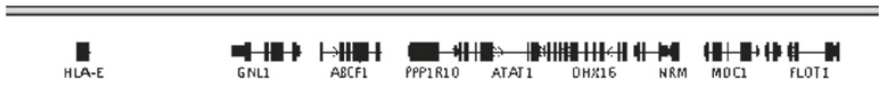

(b)
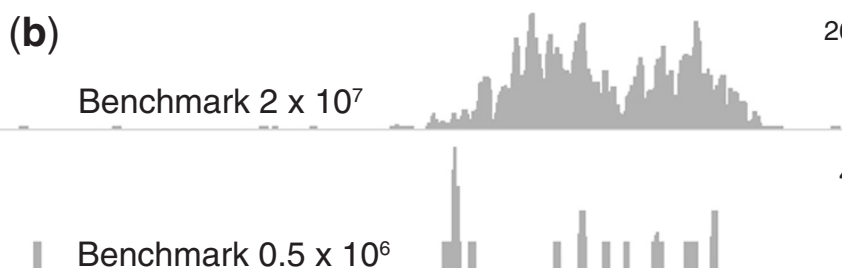

| 29
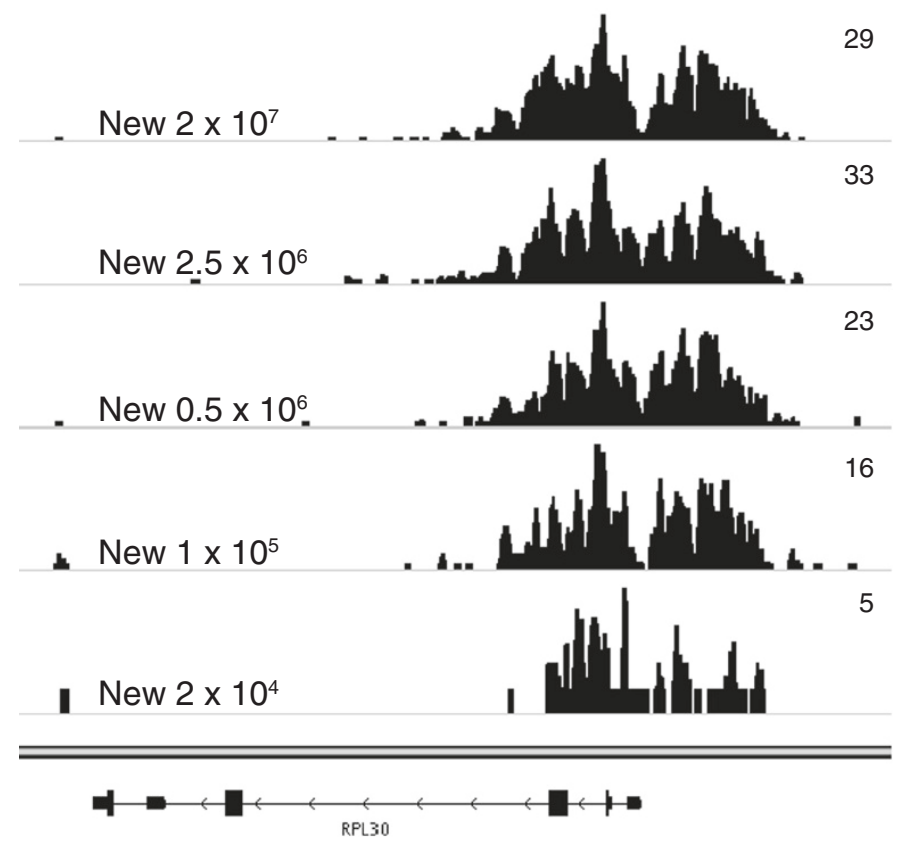

(c)

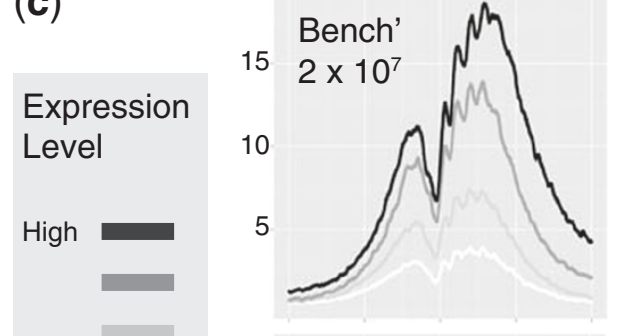

Low

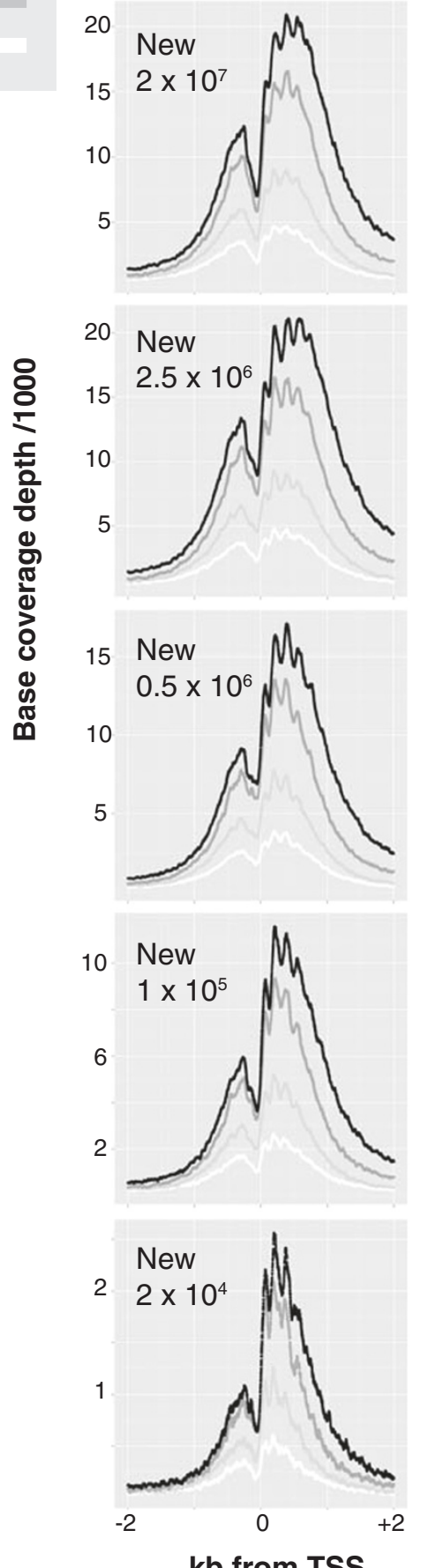

kb from TSS 
(See figure on previous page.)

Figure 2 H3K4me3 peaks are found at promoters, where peak heights parallel gene expression levels. (a) $330 \mathrm{~kb}$ section of the gene-dense major histocompatibility complex (MHC) visualised in the Integrative Genomics Viewer [25]. Tracks display read depth for benchmark (gray) and new (black) ChIP methods at decreasing input cell numbers. Maximum read depth over the displayed area is indicated on the right of each track. Only uniquely mapping, non-duplicate reads are displayed. (b) $8 \mathrm{~kb}$ region showing H3K4me3 signal over the promoter of the RPL30 gene. (c) Sequence coverage over transcription start sites (TSS). Coverage is displayed as a function of gene expression, with genes divided into quartiles based on expression level.

$1 \times 10^{5}$ cells / IP, where $85 \%$ of peaks could still be detected. As expected from the reduced number of peaks called, sensitivity fell in the lowest cell number sample to $70 \%$. Peaks were not lost randomly with reduced cell numbers. Rather, the same peaks were affected in each sample, with the preferential loss of those with lowest significance (fewest reads) evident as cell numbers were reduced.

Importantly, peak position was not adversely affected by lowering cell number. As can be seen in Figure 3b, peak overlaps between the benchmark and other datasets were close to $100 \%$ (i.e. not partially overlapping, or within an arbitrarily chosen window size). However, peak width was reduced in the lowest cell number samples (Figure 3c). Whilst every effort was made to ensure similar MNase digestion between the samples, we cannot exclude that the narrower peaks seen with lower cell numbers are due to increased digestion in these samples. However, the effect may be entirely explained by the lower number of reads available for peak calling in these samples. We are currently unable to separate the two possibilities.

In addition, extra peaks not present in the benchmark dataset were used to calculate a measure of specificity (Table 1). Specificity was not affected by scaling down cell numbers, with all datasets having greater than $90 \%$ of called peaks "on target" when comparing to the benchmark. It should be noted that this assumes that the dataset of Zhao and colleagues represents a gold standard and that additional peaks are false positives, which is not necessarily the case. Comparing the signal intensity of peaks at each location revealed a strong correlation, which deteriorated at the lowest cell number of $2 \times 10^{4}$ cells per IP (Figure 3d).

To demonstrate the application of the new method to other histone modifications, the transcriptionally repressive H3K27me3 mark was also examined. Figure 4a shows the ChIP-seq profile of H3K4me3 and H3K27me3 generated from 100,000 cells / IP at the active STAT4 and inactive $M Y O 1 B$ loci (a comparable image can be found in the paper of Barski et al., [20]). The mutually exclusive nature of H3K4 and H3K27 trimethylation is clearly visible in these profiles.

To assess the reproducibility of data generated with the new method, three independent chromatin extracts were prepared from frozen primary $\mathrm{CD}^{+}$lymphocytes, from which H3K4me3 and H3K27me3 ChIP-seq profiles were generated using 100,000 and 20,000 cells per IP. A heatmap display allowing comparison of the read depths across all 12 samples is presented in Figure $4 \mathrm{~b}$, and shows the similarity of the replicate samples across an $8 \mathrm{Mb}$ chromosomal section. The mutually-exclusive nature of the two histone modifications is also clearly visible at this scale. A quantitative measure of pairwise ChIP signal intensities (Pearson/s correlation coefficient) is shown for selected replicate pairs in Figure $4 \mathrm{c}-\mathrm{f}$, and a complete matrix of correlation coefficients is provided as Table 2. Correlation coefficients are high for both H3K4me3 and H3K27me3 datasets from 100,000 cells/ IP, and drop when 20,000 cells were used, supporting our earlier observations that the sensitivity of the technique is adversely affected at this low level of input material. The mutually exclusive nature of $\mathrm{H} 3 \mathrm{~K} 4$ and H3K27 trimethylation is confirmed here by the negative

Table 1 Peak calling, sensitivity (detection of peaks called in the benchmark) and specificity (off-target peaks not present in the benchmark)

\begin{tabular}{|c|c|c|c|c|c|c|}
\hline \multirow[t]{2}{*}{ Protocol \& cell number / ChIP } & Bench' $^{\prime}$ & New & New & New & New & New \\
\hline & $2 \times 10^{7}$ & $2 \times 10^{7}$ & $2.5 \times 10^{6}$ & $5 \times 10^{5}$ & $1 \times 10^{5}$ & $2 \times 10^{4}$ \\
\hline Total number reads & 13402262 & 13798839 & 14136243 & 16693519 & 15601563 & 56904707 \\
\hline Number unique, non-duplicate reads & 6011891 & 70330709 & 5794519 & 3463886 & 2423126 & 661591 \\
\hline \multirow[t]{2}{*}{ Number of peaks called (fraction relative to benchmark) } & 16545 & 16244 & 17054 & 15636 & 14771 & 12296 \\
\hline & $(1)$ & $(0.98)$ & $(1.03)$ & $(0.95)$ & $(0.89)$ & $(0.74)$ \\
\hline Sensitivity relative to benchmark & 1 & 0.93 & 0.96 & 0.89 & 0.85 & 0.69 \\
\hline Specificity relative to benchmark & 1 & 0.94 & 0.93 & 0.95 & 0.97 & 0.98 \\
\hline
\end{tabular}

Peaks were called using MACS, allowing no ambiguously mapping or duplicate reads. 
correlations seen when comparing these datasets. Four ChIP-seq datasets from the ENCODE project [27] have been included for comparison (two replicates each of H3K4me3 and H3K27me3 from the lymphoblastoid cell line GM12878 [28]), and show similar or poorer correlation coefficients than the datasets generated here from 100,000 cells / IP (Table 2).

Finally, to demonstrate the utility of the method using primary cell isolates, we applied it to cell samples from three pairs of human monozygotic twins. Using purified and live-frozen $\mathrm{CD}^{+}$and $\mathrm{CD}^{+}$lymphocytes (in the range of 365-500,000 cells per IP), H3K4 trimethylation profiles were prepared from each individual. A rudimentary comparison of peak calling in the three twin pairs is included here to demonstrate application of the method to primary cell isolates (Table 3). Peaks were considered concordant between a twin pair if peaks called in both individuals overlapped. Peak concordance ranged from 82-94\% in $\mathrm{CD}^{+}$cells and $73-78 \%$ in $\mathrm{CD}^{+}$lymphocytes. A more complete analysis of several twin pairs, aimed at identifying differential methylation between twins, is outside the scope of this manuscript and will be presented elsewhere.

\section{Discussion}

In this study we have developed and employed a rapid $\mathrm{N}$-ChIP technique applicable to small cell numbers, which functions well down to 100,000 cells / IP. Whilst this limit is higher than the requirements reported using alternative HTS library preparation methods [11-13], it minimizes the use of nucleic acid amplification and associated risk of bias in the data. As the first N-ChIP protocol tailored to low cell numbers, it therefore offers an attractive alternative method to map the genome-wide distribution of histone modifications. The success of the method using standard HTS library preparation techniques may reflect the reported higher efficiency of N-chip relative to X-ChIP [15]. We would also like to stress the importance of selecting specific antisera, such as using a peptide array as employed here, to the success of this or any ChIP protocol using low input cell numbers. We have demonstrated the application of this method to immuno-purified $\mathrm{CD}_{4}^{+}$and $\mathrm{CD}^{+}$primary lymphocytes, thus avoiding the need for cell culture, which risks altering epigenetic modifications. Nonetheless, the study highlights the need for careful monitoring of sequence read mapping in the analysis of ChIP-seq data from limited cell numbers, to identify sources of wasted reads and ensure sufficient coverage for reliable peak calling.

As demonstrated here, PCR amplification from limited ChIP input material led to a reduction of mappable and unique reads through losses to amplification artifacts and duplicate molecules, which must be removed for reliable peak calling. The accumulation of such undesirable amplification artifacts in alternative techniques such as LinDA and nano-ChIP-seq [11-13] has not been adequately addressed to date, and requires further examination. Notably, the method presented here entails fewer amplification cycles than these other techniques. The introduction of bias by genome-amplification techniques such as linker-mediated PCR and WGA have been documented [29]. It is therefore desirable to minimise the number of amplification cycles whenever possible. It is likely that the levels of PCR artifacts could be reduced by employing alternative amplification conditions, or through the use of alternative polymerases.

It has been shown that both MNase digestion and sonication demonstrate some DNA cleavage sequence preference, which necessitates the use of control digestion / sonication of naked DNA when interpreting HTS data with the aim of identifying nucleosome positions or regions of high accessibility such as promoters [30,31]. The existence of favourable cleavage sites will inevitably lead to a number of "duplicate" reads that are in fact biological in origin rather than amplification artifacts. This effect is likely to be more pronounced in MNase digested chromatin, as the preferential digestion of MNase in inter-nucleosomal linker regions further restricts genomic cleavage sites. It is therefore possible that the levels of duplication seen here will not be so high when using formaldehyde cross-linked chromatin, although this remains to be tested. Using the data presented here, inclusion of duplicate reads led to the appearance of many non-specific peaks, so it remains challenging to differentiate genuine biological duplicate reads from those arising as the result of the PCR process. The percentage of duplicate reads derived from PCR could be further reduced by employing paired-end reads rather than the single reads typically used, as here, for ChIP-seq analysis. Only in the situation where molecules have exactly the same length and genomic position, would paired reads be counted as a duplicate. Alternatively, utilizing multiple combinations of indexed adapters, termed digital sequencing [32], would allow the differentiation of biological and PCR-derived duplicate reads.

In addition to the problems of amplification artifacts and duplicate reads, ChIP-seq from limiting cell numbers is challenging due to the reduced complexity of immunoprecipitated material recovered. In the case of $\mathrm{CD}^{+}$cells presented here, sensitivity and reproducibility were not badly affected down to 100,000 cells / IP, but below this were reduced, although useful results were nonetheless obtained (70\% of peaks detected by benchmark method). Further improvements are likely possible by increasing the efficiency of immunoprecipitation, DNA purification, and sequencing library generation methods. 


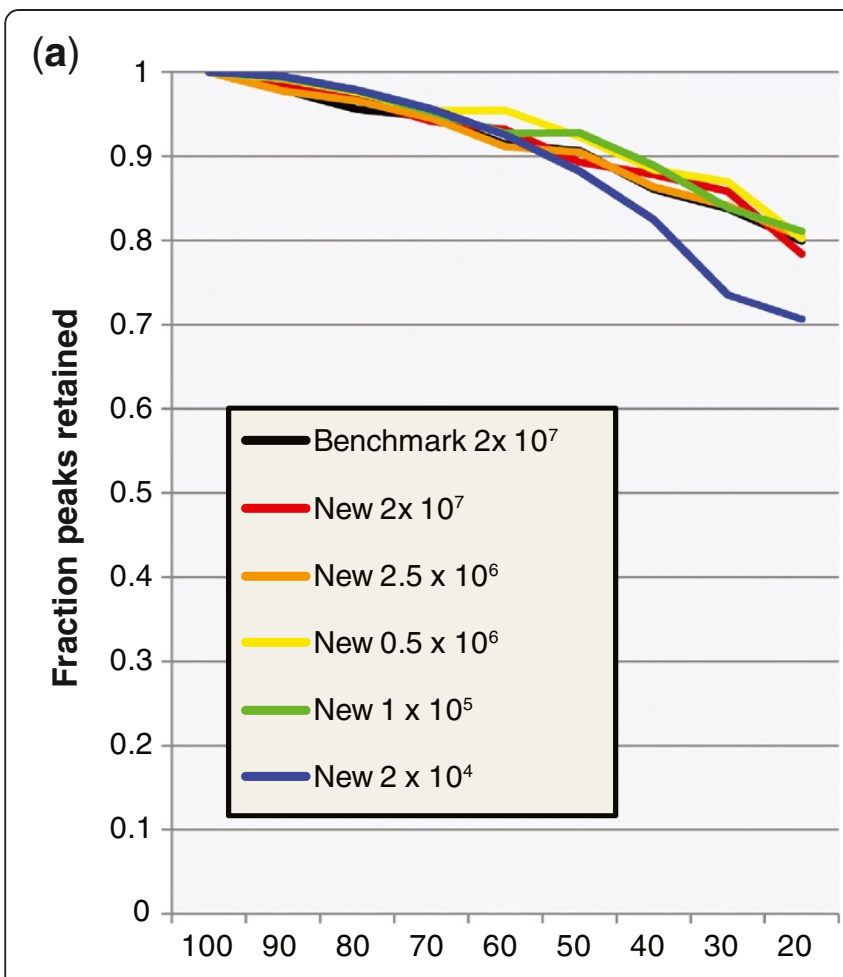

Percent reads used to call peaks

(c)

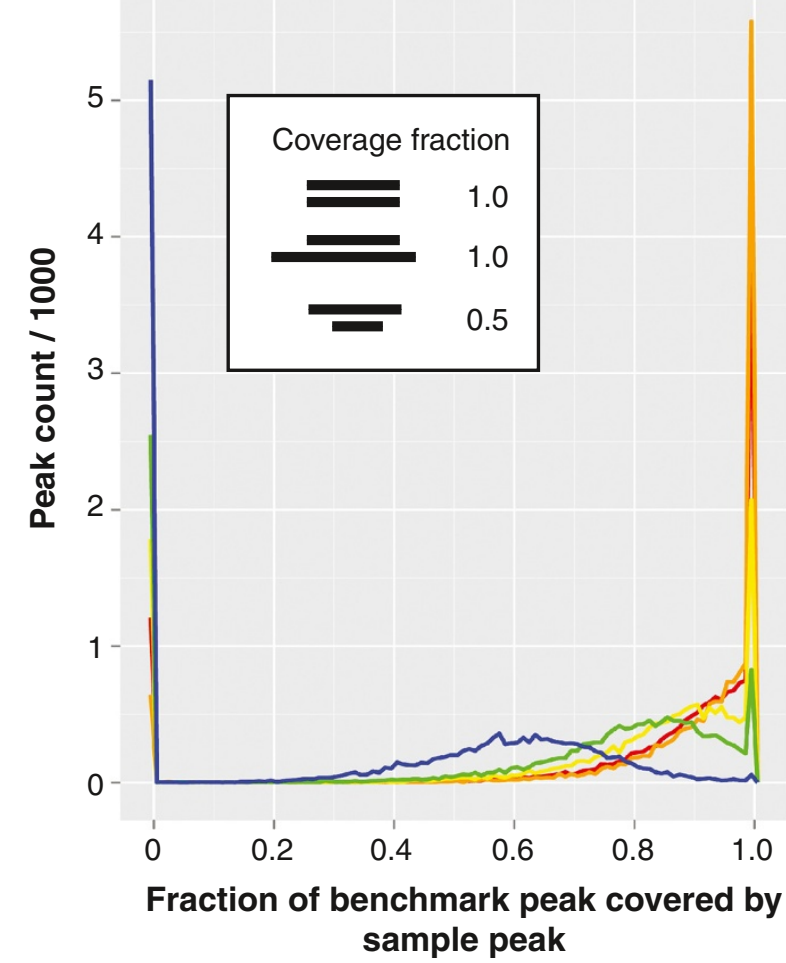

Figure $\mathbf{3}$ (See legend on next page.) (b)

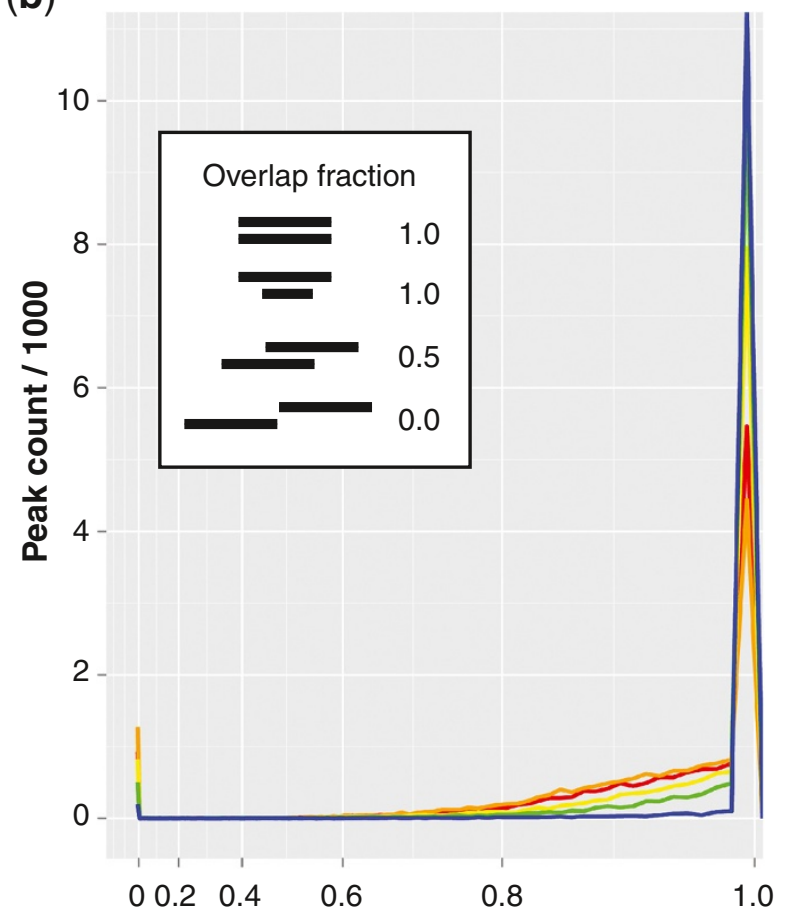

Fraction of sample peak overlapping benchmark peak

(d)

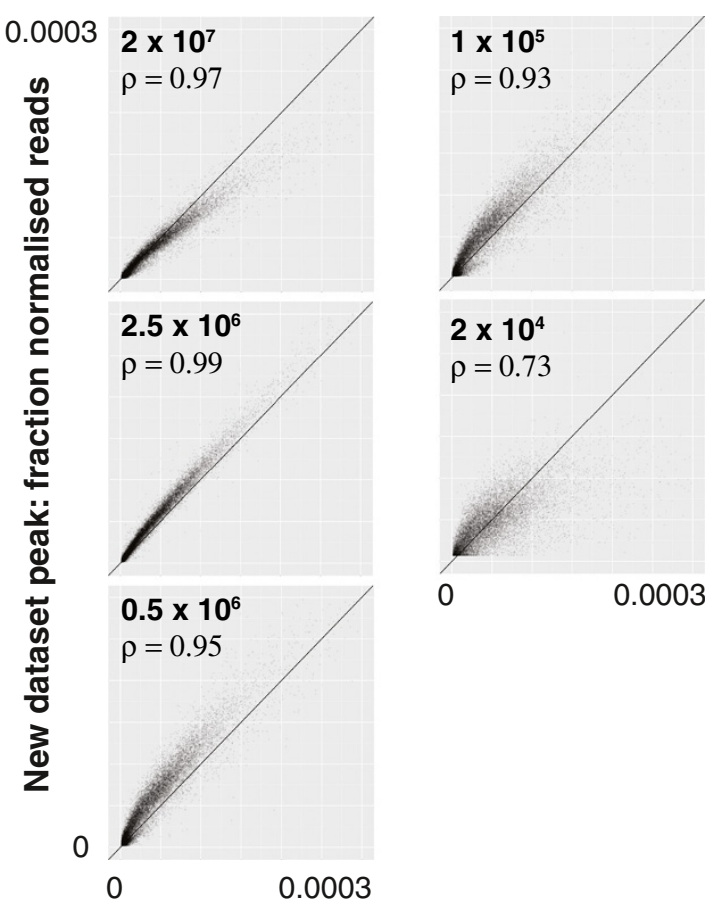

Benchmark peak: fraction normalised reads 
(See figure on previous page.)

Figure 3 Saturation, sensitivity and correlation of peak calling with decreasing cell number. (a) Saturation of peak calling as reads are randomly discarded. Peaks were called using only unique non-duplicated reads. (b) Overlap of called peaks in the different datasets with benchmark dataset peaks. Inset diagram defines examples of full or partial peak overlap, with the upper bar in each case representing the benchmark. Colours as in panel a. (c) Coverage of benchmark peaks by peaks in other datasets. Colours as in panel a. Inset shows examples of coverage, with upper bar in each case representing the benchmark. (d) Correlation of peak heights between benchmark and new sample datasets. Spearman correlation coefficients $(\rho)$ are given. Only peaks overlapping a benchmark peak were included in this analysis. The number of reads in a given peak was normalised to the total number of reads (uniquely mapping non-duplicated) in the sample.

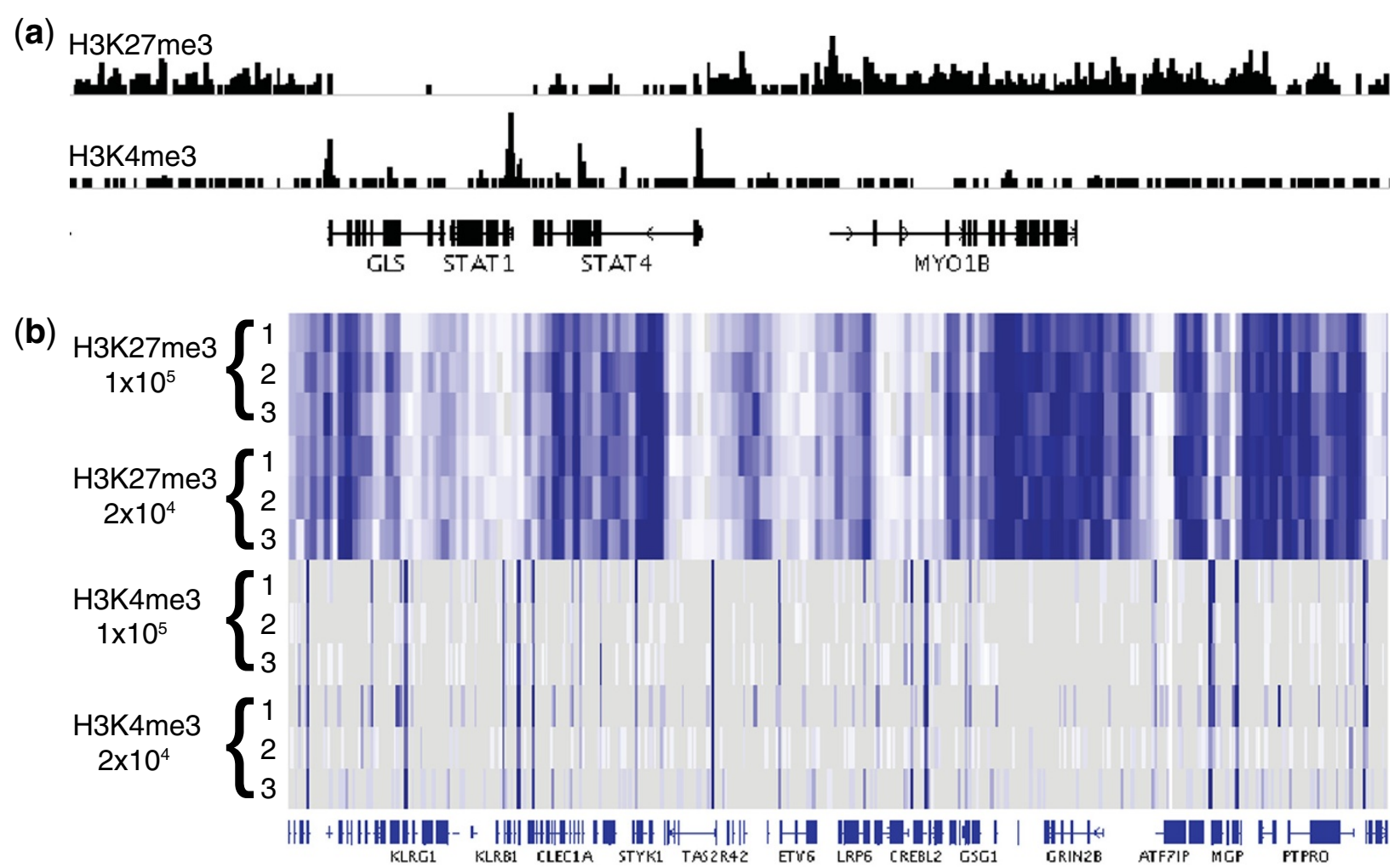

(c)

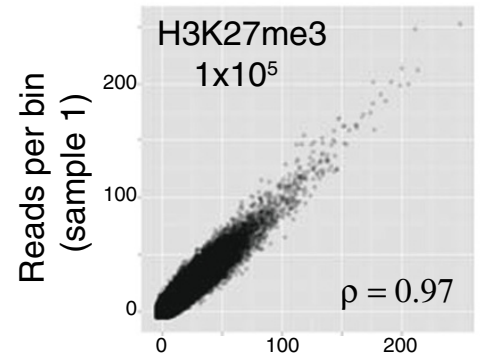

(d)

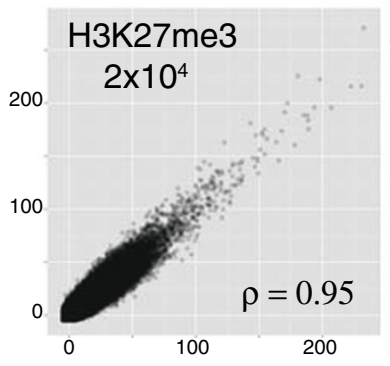

(e)

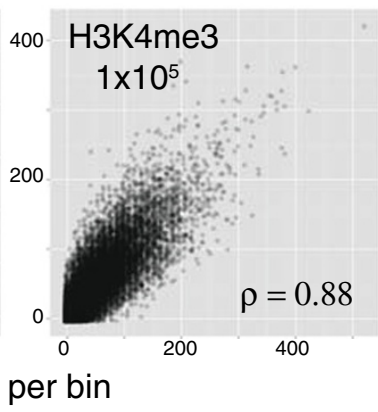

(f)

(sample 2)

Figure 4 Reproducibility of H3K4me3 and H3K27me3 ChIP-seq with the new method. (a) $1 \mathrm{Mb}$ region of chromosome 2 containing the transcriptionally active STAT1 / 4 and inactive MYO1B loci, visualised in the IGV genome browser. (b) Heatmap display in IGV genome browser showing triplicate ChIP signals over an $8 \mathrm{Mb}$ region on chromosome 12. H3K27me3 and H3K4me3 signals are shown for 50 and $10 \mathrm{~kb}$ window sizes respectively. (c-f) Genome-wide pairwise correlations of read depth in 50 kb (H3K27me3) and $10 \mathrm{~kb}$ (H3K4me3) bins for selected replicate samples. Pearson correlation coefficients are given for each comparison. Read depth per bin was normalised to the total number of uniquely mapping reads per sample (reads per bin per million uniquely mapped reads). 
Table 2 Genome-wide pairwise correlation coefficients of replicate ChIP experiments

\begin{tabular}{|c|c|c|c|c|c|c|c|c|c|c|c|c|c|c|c|c|}
\hline & GM & GM & GM & GM & H3 & $\mathrm{H} 3$ & $\mathrm{H3}$ & H3 & $\mathrm{H3}$ & $\mathrm{H} 3$ & $\mathrm{H} 3$ & $\mathrm{H3}$ & $\mathrm{H3}$ & H3 & $\mathrm{H} 3$ & H3 \\
\hline & H3 & H3 & H3 & $\mathrm{H3}$ & K27 & K27 & K27 & K27 & K27 & K27 & K4 & K4 & K4 & K4 & K4 & K4 \\
\hline & $\mathrm{K} 27$ & K27 & K4 & K4 & me3 & me3 & me3 & me3 & me3 & me3 & me3 & me3 & me3 & me3 & me3 & me3 \\
\hline & me3 & me3 & me3 & me3 & $100 \mathrm{k}$ & $100 \mathrm{k}$ & $100 \mathrm{k}$ & $20 \mathrm{k}$ & $20 \mathrm{k}$ & $20 k$ & $100 \mathrm{k}$ & $100 \mathrm{k}$ & $100 k$ & $20 k$ & $20 k$ & $20 \mathrm{k}$ \\
\hline & R1 & R2 & R1 & R2 & R1 & R2 & R3 & R1 & R2 & R3 & R1 & R2 & R3 & R1 & $\mathrm{R} 2$ & R3 \\
\hline GM H3K27 me3 R1 & 1.00 & 0.86 & 0.07 & -0.02 & 0.70 & 0.70 & 0.70 & 0.70 & 0.69 & 0.69 & -0.05 & -0.05 & -0.05 & 0.01 & 0.02 & 0.06 \\
\hline GM H3K27 me3 R2 & 0.86 & 1.00 & 0.17 & 0.16 & 0.74 & 0.70 & 0.72 & 0.72 & 0.73 & 0.70 & 0.04 & 0.05 & 0.05 & 0.09 & 0.10 & 0.12 \\
\hline GM H3K4 me3 R1 & 0.07 & 0.17 & 1.00 & 0.95 & 0.01 & 0.02 & 0.01 & 0.02 & 0.02 & 0.03 & 0.68 & 0.67 & 0.69 & 0.57 & 0.63 & 0.51 \\
\hline GM H3K4 me3 R2 & -0.02 & 0.16 & 0.95 & 1.00 & 0.00 & -0.01 & -0.01 & 0.00 & 0.01 & 0.00 & 0.72 & 0.71 & 0.74 & 0.59 & 0.64 & 0.49 \\
\hline H3K27me3 100k R1 & 0.70 & 0.74 & 0.01 & 0.00 & 1.00 & 0.95 & 0.97 & 0.96 & 0.96 & 0.94 & -0.09 & -0.09 & -0.09 & -0.06 & -0.06 & -0.03 \\
\hline H3K27me3 100k R2 & 0.70 & 0.70 & 0.02 & -0.01 & 0.95 & 1.00 & 0.94 & 0.94 & 0.93 & 0.92 & -0.09 & -0.09 & -0.09 & -0.05 & -0.05 & -0.01 \\
\hline H3K27me3 100k R3 & 0.70 & 0.72 & 0.01 & -0.01 & 0.97 & 0.94 & 1.00 & 0.95 & 0.95 & 0.93 & -0.09 & -0.09 & -0.09 & -0.05 & -0.05 & -0.02 \\
\hline H3K27me3 20k R1 & 0.70 & 0.72 & 0.02 & 0.00 & 0.96 & 0.94 & 0.95 & 1.00 & 0.95 & 0.93 & -0.09 & -0.09 & -0.09 & -0.05 & -0.05 & -0.02 \\
\hline H3K27me3 20k R2 & 0.69 & 0.73 & 0.02 & 0.01 & 0.96 & 0.93 & 0.95 & 0.95 & 1.00 & 0.93 & -0.08 & -0.08 & -0.08 & -0.04 & -0.04 & -0.01 \\
\hline H3K27me3 20k R3 & 0.69 & 0.70 & 0.03 & 0.00 & 0.94 & 0.92 & 0.93 & 0.93 & 0.93 & 1.00 & -0.08 & -0.09 & -0.09 & -0.04 & -0.04 & 0.00 \\
\hline H3K4me3 100k R1 & -0.05 & 0.04 & 0.68 & 0.72 & -0.09 & -0.09 & -0.09 & -0.09 & -0.08 & -0.08 & 1.00 & 0.86 & 0.88 & 0.70 & 0.76 & 0.59 \\
\hline H3K4me3 100k R2 & -0.05 & 0.05 & 0.67 & 0.71 & -0.09 & -0.09 & -0.09 & -0.09 & -0.08 & -0.09 & 0.86 & 1.00 & 0.87 & 0.69 & 0.76 & 0.59 \\
\hline H3K4me3 100k R3 & -0.05 & 0.05 & 0.69 & 0.74 & -0.09 & -0.09 & -0.09 & -0.09 & -0.08 & -0.09 & 0.88 & 0.87 & 1.00 & 0.70 & 0.77 & 0.58 \\
\hline H3K4me3 20k R1 & 0.01 & 0.09 & 0.57 & 0.59 & -0.06 & -0.05 & -0.05 & -0.05 & -0.04 & -0.04 & 0.70 & 0.69 & 0.70 & 1.00 & 0.66 & 0.56 \\
\hline H3K4me3 20k R2 & 0.02 & 0.10 & 0.63 & 0.64 & -0.06 & -0.05 & -0.05 & -0.05 & -0.04 & -0.04 & 0.76 & 0.76 & 0.77 & 0.66 & 1.00 & 0.60 \\
\hline H3K4me3 20k R3 & 0.06 & 0.12 & 0.51 & 0.49 & -0.03 & -0.01 & -0.02 & -0.02 & -0.01 & 0.00 & 0.59 & 0.59 & 0.58 & 0.56 & 0.60 & 1.00 \\
\hline
\end{tabular}

The future adoption of epigenetic analyses in research and diagnostic procedures will require techniques that allow analysis of specific cell types, sub-populations and small biopsy samples. For example, the N-ChIP technique has been optimised for analysis of the Schistosoma parasite [33], and techniques have been developed for ChIP from particular structures such as the hippocampus [34]. We have demonstrated here the application of the enhanced method to lymphocytes isolated from human twins. The cells used for study were enriched using antibody-driven magnetic cell sorting, derived from a starting material of $10-20 \mathrm{mls}$ blood. It would not have been possible to study such small, easily obtained biological samples using previously published N-ChIP procedures. Further

Table 3 ChIP-seq from primary cells isolated from human monozygotic twins

\begin{tabular}{|c|c|c|c|c|c|c|}
\hline \multirow{2}{*}{$\begin{array}{l}\text { Twin Pair } \\
\text { CD4 }^{+} \text {cells }\end{array}$} & \multicolumn{2}{|c|}{ Twin pair 1} & \multicolumn{2}{|c|}{ Twin pair 2} & \multicolumn{2}{|c|}{ Twin pair 3} \\
\hline & & & & & & \\
\hline Cell no. / IP & $5 \times 10^{5}$ & $5 \times 10^{5}$ & $5 \times 10^{5}$ & $5 \times 10^{5}$ & $5 \times 10^{5}$ & $5 \times 10^{5}$ \\
\hline No. reads & 35244517 & 44255574 & 40644508 & 45738891 & 38332819 & 29484478 \\
\hline No. unique, nonduplicate reads & 3978339 & 6524094 & 3316312 & 3340926 & 3130804 & 1167824 \\
\hline No. Peaks called & 14828 & 12622 & 15598 & 15731 & 15825 & 13719 \\
\hline No. overlapping peaks (\%) & \multicolumn{2}{|c|}{12457 (82\%) } & \multicolumn{2}{|c|}{$14833(94 \%)$} & \multicolumn{2}{|c|}{$13091(83 \%)$} \\
\hline \multicolumn{7}{|l|}{$\mathrm{CD8}^{+}$cells } \\
\hline Cell no. / IP & $4.3 \times 10^{5}$ & $3.7 \times 10^{5}$ & $4.7 \times 10^{5}$ & $5 \times 10^{5}$ & $3.8 \times 10^{5}$ & $4.2 \times 10^{5}$ \\
\hline No. reads & 45245361 & 35681254 & 38455042 & 35257788 & 34505357 & 41729689 \\
\hline No. unique, nonduplicate reads & 18996309 & 4778486 & 12403566 & 6654203 & 5840308 & 4438574 \\
\hline No. Peaks called & 17704 & 18720 & 20728 & 18145 & 18743 & 20899 \\
\hline No. overlapping peaks (\%) & \multicolumn{2}{|c|}{$14578(78 \%)$} & \multicolumn{2}{|c|}{$18828(76 \%)$} & \multicolumn{2}{|c|}{$15312(73 \%)$} \\
\hline
\end{tabular}

Peaks were called using MACS, allowing no ambiguously mapping or duplicate reads. Peaks with $p$-values $>1 \times 10^{-10}$ were excluded from analysis. Numbers of overlapping peaks were counted, and expressed as a percentage of the highest peak count for the twin pair. 
improvements in both ChIP protocols and HTS library preparation methods, such as single molecule sequencing [35] promise to increase the possibilities for epigenetic studies from these and other challenging samples.

\section{Conclusions}

Using an enhanced native ChIP-seq method, we have detailed known but hitherto uncharacterised problems of performing ChIP-seq from limited cell numbers. Using our method, high quality results were obtained from 200,000 cells starting material (using 100,000 cells per IP), increasing possibilities for the study of rare cell populations and biopsy samples without the need for cell culture. It was possible to generate results from as low as 20,000 cells per IP, but at a cost to sensitivity, where only $70 \%$ of known peaks could be detected, so we declare the limit of this method to currently require 100,000 cells per IP. This method offers an alternative to other previously published methods for low cell number ChIP-seq that entails fewer cycles of amplification with associated risk of data bias. Nonetheless, as cell numbers are reduced, the amount and complexity of immunoprecipitated material is reduced. Amplification of this material leads to a corresponding rise in PCR duplicates and unmapped reads, which may necessitate costly additional sequencing, an effect that requires further characterisation in comparable methods. Additional improvements in the efficiency of immunoprecipitation and HTS library generation techniques are desirable to bring us closer to the goal of single cell analysis.

\section{Methods}

\section{Isolation of lymphocyte sub-populations \& cell culture}

Peripheral blood mononuclear cells (PBMCs) were isolated from an anonymous human blood donor using Lymphoprep reagent (Axis-Shield plc, Dundee, UK). CD8 ${ }^{+}$ and $\mathrm{CD}^{+}$cells were sequentially isolated using an AutoMACS Pro separator (Miltenyi Biotec, Köln, Germany) by positive and negative isolation, respectively. Cells were live-frozen in freezing medium (20\% v/v DMSO; $80 \% \mathrm{v} / \mathrm{v}$ Fetal Calf Serum (Lonza, Basel, Switzerland)) in aliquots. For experiments requiring up to $4 \times 10^{7}$ cells per chromatin preparation, two million thawed $\mathrm{CD} 4^{+}$cells were activated using Human T-Expander CD3/CD28 (Invitrogen, Carlsbad, CA) according to manufacturers instructions and cells were cultured in X-VIVO media (Lonza) supplemented with Interleukin II $(10 \mathrm{ng} / \mathrm{ul})$ for a period of two weeks at $37^{\circ} \mathrm{C}$ in a humidified incubator under $5 \% \mathrm{CO}_{2}$. For experiments with human twin cell populations, thawed $\mathrm{CD}^{+}$or $\mathrm{CD}^{+}$cells were used directly as input into the ChIP procedure as detailed below.

\section{Antibody selection}

Polyclonal antisera against H3K4me3 (Diagenode s.a., Liège, Belgium; Cat. \# pAb-003-050; Lot \# A2-002P) and
H3K27me3 (Upstate Biotechnology, Temecula, CA; Cat. \# 07-449; Lot \# DAM1387952) were selected after screening several commercially available antisera for specificity using a custom histone peptide microarray (JPT Peptide Technologies Gmbh, Berlin, Germany). Arrays were spotted with 156 different selected peptides of length 13 residues, representing covalently modified Nterminal tails of $\mathrm{H} 3$ and $\mathrm{H} 4$ (details available upon request). Results of specificity testing are available in the HisMAD database [36].

\section{Chromatin immunoprecipitation}

The method of Zhao and colleagues [21] was followed and used as a benchmark to which our new method was compared. The new method differs from the benchmark in the following ways: (i) The requirement for dialysis was removed by instead diluting chromatin in a concentrated immunoprecipitation buffer, allowing faster handling and reduced material loss. (ii) Sonication was performed in a Bioruptor sonicator (Diagenode, Liège, Belgium) using TPX plasticware to allow sonication of small volumes with minimal sample loss and eliminate potential sources of contamination. (iii) Control of MNase Digestion was performed on a 2100 Bioanalyzer (Agilent Technologies, Santa Clara, CA) to allow visualisation of much lower DNA amounts. (iv) Illumina library preparation was altered to retain nucleosome-sized fragments upon size selection, and (v) replace columnbased cleanup steps with SPRI-beads (Beckman Coulter, Beverly, MA) to retain more DNA at every step.

The method is summarised below. In addition, a detailed step-by-step method is provided as Additional file 1 . The method presented here is specifically tailored for sequencing on Illumina technology, but should be easily adaptable to other sequencing platforms. Cultured $\mathrm{CD}_{4}{ }^{+}$cells were counted, then harvested by centrifugation at $1500 \times \mathrm{G}$ for $5 \mathrm{mins}$ at room temp and washed in PBS. Cells were divided at this stage into separate tubes according to the titration of starting cell numbers required. Cell pellets were then resuspended in digestion buffer (50 mM Tris- $\mathrm{HCl}, \mathrm{pH} 8.0 ; 1 \mathrm{mM} \mathrm{CaCl} 2 ; 0.2 \%$ Triton X-100) at room temperature supplemented with protease inhibitors. Because of impracticalities associated with handling cell numbers spanning 3 orders of magnitude, it was not possible to use exactly the same volumes and concentrations for all steps, and these differences have been summarised in Table 4. Micrococcal nuclease (USB, Cleveland, $\mathrm{OH}$ ) was added to a concentration of 0.19 units per $1 \times 10^{7}$ cells, and incubated for 5 mins at $37^{\circ} \mathrm{C}$. Digestion was terminated by the addition of 0.1 volumes of stop solution (110 mM Tris $\mathrm{pH} 8.0 ; 55 \mathrm{mM}$ EDTA) and samples transferred to ice. Samples were then subjected to brief sonication in a Bioruptor (Diagenode) for 60 seconds, on high power with no pulsing in 
Table 4 Variable parameters applied to chromatin from different starting cell numbers

\begin{tabular}{|c|c|c|c|c|c|c|}
\hline \multirow{2}{*}{$\begin{array}{l}\text { Protocol \& starting cell } \\
\text { number }\end{array}$} & Benchmark & New & New & New & New & New \\
\hline & $4 \times 10^{7}$ & $4 \times 10^{7}$ & $5 \times 10^{6}$ & $1 \times 10^{6}$ & $2 \times 10^{5}$ & $4 \times 10^{4}$ \\
\hline Cells per IP & $2 \times 10^{7}$ & $2 \times 10^{7}$ & $2.5 \times 10^{6}$ & $5 \times 10^{5}$ & $1 \times 10^{5}$ & $2 \times 10^{4}$ \\
\hline \multirow{2}{*}{$\begin{array}{l}\text { MNase digestion } \\
\text { volume (cells / ml) }\end{array}$} & $1500 \mu \mathrm{l}$ & $1500 \mu \mathrm{l}$ & $500 \mu \mathrm{l}$ & $100 \mu \mathrm{l}$ & $20 \mu \mathrm{l}$ & $20 \mu \mathrm{l}$ \\
\hline & $\left(2.7 \times 10^{7} / \mathrm{ml}\right)$ & $\left(2.7 \times 10^{7} / \mathrm{ml}\right)$ & $\left(1 \times 10^{7} / \mathrm{ml}\right)$ & $\left(1 \times 10^{7} / \mathrm{ml}\right)$ & $\left(1 \times 10^{7} / \mathrm{ml}\right)$ & $\left(2 \times 10^{6} / \mathrm{ml}\right)$ \\
\hline \multirow[t]{2}{*}{ IP volume } & $750 \mu \mathrm{l}$ & $1500 \mu \mathrm{l}$ & $500 \mu \mathrm{l}$ & $100 \mu \mathrm{l}$ & $100 \mu \mathrm{l}$ & $100 \mu \mathrm{l}$ \\
\hline & (in $1.5 \mathrm{ml}$ tube) & (in $2 \mathrm{ml}$ tube) & (in $1.5 \mathrm{ml}$ tube) & (in $0.2 \mathrm{ml} \mathrm{PCR} \mathrm{tube)}$ & (in $0.2 \mathrm{ml} \mathrm{PCR} \mathrm{tube)}$ & (in $0.2 \mathrm{ml} \mathrm{PCR} \mathrm{tube)}$ \\
\hline $\begin{array}{l}\text { Protein } A / G \text { bead } \\
\text { volume for preclearing / IP }\end{array}$ & $50 \mu \mathrm{l}$ & $50 \mu l$ & $50 \mu l$ & $10 \mu \mathrm{l}$ & $10 \mu \mathrm{l}$ & $10 \mu \mathrm{l}$ \\
\hline Antibody amount / IP & $5 \mu \mathrm{g}$ & $5 \mu \mathrm{g}$ & $5 \mu \mathrm{g}$ & $1 \mu \mathrm{g}$ & $1 \mu \mathrm{g}$ & $1 \mu \mathrm{g}$ \\
\hline Wash buffer volumes & $1 \mathrm{ml}$ & $1 \mathrm{ml}$ & $1 \mathrm{ml}$ & $150 \mu \mathrm{l}$ & $150 \mu \mathrm{l}$ & $150 \mu \mathrm{l}$ \\
\hline
\end{tabular}

"Benchmark" refers to the protocol now published by Zhao and colleagues [21] and "new" to the method presented here.

TPX tubes (Diagenode) to assist with recovery of oligonucleosomes. Samples were then adjusted to RIPA buffer conditions by the addition of 1 volume 2xRIPA IP buffer (280 mM NaCl; $1.8 \%$ Triton X-100; $0.2 \%$ SDS; $0.2 \% \mathrm{Na}-$ Deoxycholate; $5 \mathrm{mM}$ EGTA) supplemented with protease inhibitors, and spun in a microcentrifuge at $16,000 \times \mathrm{G}$ for $15 \mathrm{mins}$ at $4^{\circ} \mathrm{C}$. Supernatants were immediately removed to fresh tubes and 10\% volume removed for DNA purification as "input". DNA was later purified from input samples by adding proteinase K (USB, Cleveland, $\mathrm{OH}$ ) to a final concentration of $0.5 \mathrm{mg} / \mathrm{ml}$ and incubating at $55^{\circ} \mathrm{C}$ for 1 hour, prior to purification over a Genomic DNA cleanup \& Concentrator column (Zymo Research Corp., Irvine, CA). The size of input DNA was then measured on a 2100 Bioanalyzer (Agilent Technologies) using high sensitivity reagents, to check that all samples had similar digestion levels and were primarily composed of mono-nucleosomes. The remaining majority of the chromatin was immediately used for immunoprecipitation. Chromatin was first precleared by the addition of a 1:1 mix of protein A and G Dynabeads (Invitrogen, Carlsbad, CA) with rotation at $4^{\circ} \mathrm{C}$ for 1 hour (see Table 4). Chromatin was then divided into two for specific IP with H3K4me3 or control IgG (see Table 4) and incubated overnight with rotation, followed by immunoprecipitation with protein A and G Dynabeads for 2 hours at $4{ }^{\circ} \mathrm{C}$. Beads were immobilised on magnetic racks and the supernatants discarded, after which the beads were washed five times with RIPA buffer (10 mM Tris pH 8.0; 1 mM EDTA; $140 \mathrm{mM} \mathrm{NaCl} ; 1 \%$ Triton X-100; $0.1 \%$ SDS; $0.1 \% \mathrm{Na}-$ Deoxycholate) and once with $\mathrm{LiCl}$ wash buffer $(250 \mathrm{mM}$ LiCl; $10 \mathrm{mM}$ Tris pH 8.0; 1 mM EDTA; 0.5\% Igepal CA630; $0.5 \% \mathrm{Na}$-deoxycholate). All washes were carried out for 5 mins at $4^{\circ} \mathrm{C}$ on a rotating wheel (see Table 4 for volumes) in the presence of protease inhibitors. Beads were finally rinsed with TE buffer without protease inhibitors. Beads were then resuspended in $100 \mu \mathrm{l}$ TE containing $0.5 \mathrm{mg} / \mathrm{ml}$ proteinase $\mathrm{K}$ and incubated at $55^{\circ} \mathrm{C}$ for
1 hour with shaking, prior to purification over a purification column as detailed for input DNA above, eluting in $50 \mu \mathrm{l} 5 \mathrm{mM}$ Tris buffer.

\section{Real-time PCR}

Two microliters of ChIP eluate were used per reaction to control the success of the immunoprecipitations using the following real-time PCR primer combinations: H3K4me3 positive control locus TaqMan assay (probe with $5^{\prime}$ FAM and $3^{\prime}$ blackhole quencher 1) [RPL30-F CAAGGCAAAGCGAAATTGGT; RPL30-R GCCCGTTCAGTCTCTTCGATT; RPL30-P TCTCGC TAACAACTGCCCAGCTTTGAG], negative control locus SYBR Green assay [NegC1-F ACGTACCTTA AGCCCCTGGT; NegC1-R TAGTGCCTGGAGTGAG GATG]. Primers were obtained from MWG Biotech (Ebersberg, Germany), and reactions performed using TaqMan or SYBR Green universal PCR master mixes (Applied Biosystems, Foster City, CA).

\section{Illumina library preparation}

Twenty five microliters of immunoprecipitated or input DNA (ranging from $<1 \mathrm{ng}-4 \mathrm{ng}$ DNA) was used for library preparation using Illumina (San Diego, CA) TruSeq $^{\mathrm{TM}}$ DNA Sample Preparation reagents, with ligation of $1 / 10^{\text {th }}$ the manufacturers recommended adapter amounts and agarose gel selection of DNA fragments in the $200-500$ bp size range.

\section{High-throughput sequencing}

Sequencing (36 bp single reads) was performed on an Illumina Genome Analyzer IIx using v4 cluster generation and v3 sequencing reagents, using one lane per sample. Yields varied within the range of 13.4 to 17.1 million reads passing filters per sample $(0.48-0.62 \mathrm{~Gb})$. Alternatively, for additional sequencing of a single sample (ChIP from $2 \times 10^{4}$ cells) and for all ChIP experiments using live-frozen human lymphocytes, libraries were sequenced (50 bp single-end reads) on an Illumina 
HiSeq 2000 using TruSeq v2.0 clustering and SBS sequencing reagents. Samples run on the HiSeq 2000 were indexed and run together, such that each sample obtained reads equivalent to half a lane on the flow cell.

\section{Data analysis}

Illumina GAIIx image analysis and base calling was performed using Illumina's RTA software version 1.4 and experiments performed on the Illumina HiSeq 2000 were analysed using RTA version 1.12. Reads were filtered to remove those with low base call quality using Illumina's default chastity criteria. Reads were mapped to the human reference genome (release hg18 / NCBI36) using BWA version 0.5.9 with default settings [37]. Peak calling was performed by MACS version 1.4 [26] using shift size determined by the size of sequencing library inserts, and switching off local background estimation (as recommended for histone modification peak calling in the absence of a control immunoprecipitation). Peak calling was only based on reads mapping to a single location, excluding duplicates. Manipulation of alignment and peak files was performed using samtools version 0.1.15 [38] and bedtools version 2.11.2 [39] software. We investigated the reproducibility of the protocol between different samples by dividing the genome into nonoverlapping $10 \mathrm{k}$ or $50 \mathrm{k}$ bins and then computing the number of uniquely mapping non-duplicate reads in each bin. The count of reads in each bin was then normalised for the sample's total number of uniquely mapping non-duplicate reads. In order to visualise the same data in a genome browser, we applied the count command of the igvtools utilities to the dataset of uniquely mapping non-duplicate reads and displayed the result in IGV using the heatmap setting [25].

\section{Histone methylation levels surrounding gene start positions}

Gene expression data for $\mathrm{CD}^{+} \mathrm{T}$ lymphocytes was obtained from the Gene Expression Omnibus[40] dataset GSE473 [41]. Affymetrix microarray design U133A expression data was retrieved for 10 control individuals, from which average gene expression levels were calculated and used to assign genes to highest or lowest quartile expression categories. H3K4me3 ChIP read depth was calculated $2 \mathrm{~kb}$ upstream and downstream of gene start positions.

\section{Additional file}

Additional file 1: A detailed protocol of the native ChIP-seq procedure described herein.

\section{Abbreviations}

HTS: High throughput sequencing; IP: Immunoprecipitation; ChIP: Chromatin immunoprecipitation; N-ChIP: Native chromatin immunoprecipitation;
X-ChIP: Formaldehyde cross-linked chromatin immunoprecipitation; ChIP-seq: Genome-wide analysis of ChIP employing high throughput sequencing; H3K4me3: Histone H3 lysine 4 trimethylation; MNase: Micrococcal nuclease; TSS: Transcription start site.

\section{Competing interests}

The authors declare no competing interests.

\section{Authors' contributions}

The study was designed by GDG as part of a project conceived by RL, DEU and GDG. ChIP and sequencing library preparation was performed by HSH and GDG. Donor cell samples were assimilated by $\mathrm{JH}$, and cell isolation procedures developed by KG. Antibody specificity testing was performed by TS. Data analysis was performed by TH and YS, and interpreted by TS and GDG. All authors contributed to the manuscript and approved it for publication.

\section{Acknowledgments}

We thank K. Zhao for sharing a detailed experimental protocol, upon which this work was developed. Sequencing was performed by the Norwegian High-Throughput Sequencing Centre, a national technology platform supported by the national "Functional Genomics (FUGE)" and "Infrastructure" programs of the Research Council of Norway and the Southeastern Regional Health Authorities. We thank the Protein Analysis Unit (ZfP) of Ludwig Ludwig Maximilians Universität, München, for antibody specificity testing. We would also like to thank Martin Hammerø, Heidi Nygård and Rune Moe for assistance with sample selection and collection.

\section{Author details}

${ }^{1}$ Department of Medical Genetics, Oslo University Hospital, Oslo, Norway. ${ }^{2}$ Ludwig Maximilians Universität, Adolf Butenandt Institut, Lehrstuhl für Molekularbiologie, Schillerstraße 44, München 80336, Germany. ${ }^{3}$ Department of Medical Genetics, Oslo University Hospital and University of Oslo, Oslo, Norway. ${ }^{4}$ Division of Epidemiology, Norwegian Institute of Public Health, Oslo, Norway.

Received: 30 April 2012 Accepted: 5 November 2012

Published: 21 November 2012

\section{References}

1. Ho JW, Bishop E, Karchenko PV, Negre N, White KP, Park PJ: ChIP-chip versus ChIP-seq: lessons for experimental design and data analysis. BMC Genomics 2011, 12:134.

2. O'Neill LP, VerMilyea MD, Turner BM: Epigenetic characterization of the early embryo with a chromatin immunoprecipitation protocol applicable to small cell populations. Nat Genet 2006, 38:835-841.

3. Attema JL, Papathanasiou P, Forsberg EC, Xu J, Smale ST, Weissman IL: Epigenetic characterization of hematopoietic stem cell differentiation using miniChIP and bisulfite sequencing analysis. Proc Natl Acad Sci U S A 2007, 104:12371-12376.

4. Dahl JA, Collas P: Q2ChIP, a quick and quantitative chromatin immunoprecipitation assay, unravels epigenetic dynamics of developmentally regulated genes in human carcinoma cells. Stem Cells 2007, 25:1037-1046.

5. Dahl JA, Collas P: A rapid micro chromatin immunoprecipitation assay (microChIP). Nat Protoc 2008, 3:1032-1045.

6. Dahl JA, Collas P: MicroChIP-a rapid micro chromatin immunoprecipitation assay for small cell samples and biopsies. Nucleic Acids Res 2008, 36:e15

7. Sikes ML, Bradshaw JM, Ivory WT, Lunsford $J$, McMillan RE, Morrison CR A streamlined method for rapid and sensitive chromatin immunoprecipitation. J Immunol Methods 2009, 344:58-63.

8. Acevedo LG, Iniguez AL, Holster HL, Zhang $X$, Green R, Farnham PJ: Genome-scale ChIP-chip analysis using 10,000 human cells. Biotechniques 2007, 43:791-797.

9. Dahl JA, Reiner AH, Collas P: Fast genomic muChIP-chip from 1,000 cells. Genome Biol 2009, 10:R13.

10. Hitchler MJ, Rice JC: Genome-Wide Epigenetic Analysis of Human Pluripotent Stem Cells by ChIP and ChIP-Seq. Methods Mol Biol 2011 767:253-267. 
11. Adli M, Zhu J, Bernstein BE: Genome-wide chromatin maps derived from limited numbers of hematopoietic progenitors. Nat Methods 2010, 7:615-618.

12. Adli $M$, Bernstein BE: Whole-genome chromatin profiling from limited numbers of cells using nano-ChIP-seq. Nat Protoc 2011, 6:1656-1668.

13. Shankaranarayanan P, Mendoza-Parra MA, Walia M, Wang L, Li N, Trindade LM, Gronemeyer $H$ : Single-tube linear DNA amplification (LinDA) for robust ChIP-seq. Nat Methods 2011, 8:565-567.

14. O'Neill LP, Turner BM: Histone $\mathrm{H} 4$ acetylation distinguishes coding regions of the human genome from heterochromatin in a differentiation-dependent but transcription-independent manner. EMBO J 1995, 14:3946-3957

15. O'Neill LP, Turner BM: Immunoprecipitation of native chromatin: NChIP. Methods 2003, 31:76-82.

16. Thorne AW, Myers FA, Hebbes TR: Native chromatin immunoprecipitation. Methods Mol Biol 2004, 287:21-44.

17. Umlauf D, Goto Y, Feil R: Site-specific analysis of histone methylation and acetylation. Methods Mol Biol 2004, 287:99-120.

18. Gregory RI, Randall TE, Johnson CA, Khosla S, Hatada I, O'Neill LP, Turner BM, Feil R: DNA methylation is linked to deacetylation of histone $\mathrm{H} 3$, but not H4, on the imprinted genes Snrpn and U2af1-rs1. Mol Cell Biol 2001, 21:5426-5436.

19. Roca H, Franceschi RT: Analysis of transcription factor interactions in osteoblasts using competitive chromatin immunoprecipitation. Nucleic Acids Res 2008, 36:1723-1730

20. Barski A, Cuddapah S, Cui K, Roh TY, Schones DE, Wang Z, Wei G, Chepelev I, Zhao K: High-resolution profiling of histone methylations in the human genome. Cell 2007, 129:823-837.

21. Cuddapah S, Barski A, Cui K, Schones DE, Wang Z, Wei G, Zhao K: Native chromatin preparation and Illumina/Solexa library construction. Cold Spring Harb Protoc 2009, 2009:db.

22. The Genbank Database: http://www.ncbi.nlm.nih.gov/genbank/.

23. Altschul SF, Gish W, Miller W, Myers EW, Lipman DJ: Basic local alignment search tool. J Mol Biol 1990, 215:403-410.

24. Bernstein BE, Kamal M, Lindblad-Toh K, Bekiranov S, Bailey DK, Huebert DJ, McMahon S, Karlsson EK, Kulbokas EJ III, Gingeras TR, et al: Genomic maps and comparative analysis of histone modifications in human and mouse. Cell 2005, 120:169-181.

25. Robinson JT, Thorvaldsdottir H, Winckler W, Guttman M, Lander ES, Getz G, Mesirov JP: Integrative genomics viewer. Nat Biotechnol 2011, 29:24-26.

26. Zhang Y, Liu T, Meyer CA, Eeckhoute J, Johnson DS, Bernstein BE, Nusbaum C, Myers RM, Brown M, Li W, et al: Model-based analysis of ChIP-Seq (MACS). Genome Biol 2008, 9:R137.

27. Bernstein BE, Birney E, Dunham I, Green ED, Gunter C, Snyder M: An integrated encyclopedia of DNA elements in the human genome. Nature 2012, 489:57-74.

28. ENCODE Broad Histone datasets: http://hgdownload.cse.ucsc.edu/ goldenPath/hg19/encodeDCC/wgEncodeBroadHistone/.

29. Ponzielli R, Boutros PC, Katz S, Stojanova A, Hanley AP, Khosravi F, Bros C, Jurisica I, Penn LZ: Optimization of experimental design parameters for high-throughput chromatin immunoprecipitation studies. Nucleic Acids Res 2008, 36:e144.

30. Auerbach RK, Euskirchen G, Rozowsky J, Lamarre-Vincent N, Moqtaderi Z, Lefrancois P, Struhl K, Gerstein M, Snyder M: Mapping accessible chromatin regions using Sono-Seq. Proc Natl Acad Sci U S A 2009, 106:14926-14931.

31. Chung HR, Dunkel I, Heise F, Linke C, Krobitsch S, Ehrenhofer-Murray AE, Sperling SR, Vingron M: The effect of micrococcal nuclease digestion on nucleosome positioning data. PLoS One 2010, 5:e15754.

32. Shiroguchi K, Jia TZ, Sims PA, Xie XS: Digital RNA sequencing minimizes sequence-dependent bias and amplification noise with optimized single-molecule barcodes. Proc Natl Acad Sci U S A 2012, 109:1347-1352.

33. Cosseau C, Azzi A, Smith K, Freitag M, Mitta G, Grunau C: Native chromatin immunoprecipitation (N-ChIP) and ChIP-Seq of Schistosoma mansoni: Critical experimental parameters. Mol Biochem Parasitol 2009, 166:70-76.

34. Sailaja BS, Takizawa T, Meshorer E: Chromatin immunoprecipitation in mouse hippocampal cells and tissues. Methods Mol Biol 2012, 809:353-364.

35. Goren A, Ozsolak F, Shoresh N, Ku M, Adli M, Hart C, Gymrek M, Zuk O, Regev A, Milos PM, et al: Chromatin profiling by directly sequencing small quantities of immunoprecipitated DNA. Nat Methods 2010, 7:47-49.
36. Straub T: Histone Modifications Antibody Database (HisMAD). http://wpl054.bio.med.uni-muenchen.de/HISMAD/.

37. Li H, Durbin R: Fast and accurate short read alignment with Burrows-Wheeler transform. Bioinformatics 2009, 25:1754-1760.

38. Li H, Handsaker B, Wysoker A, Fennell T, Ruan J, Homer N, Marth G, Abecasis $\mathrm{G}$, Durbin R: The Sequence Alignment/Map format and SAMtools. Bioinformatics 2009, 25:2078-2079.

39. Quinlan AR, Hall IM: BEDTools: a flexible suite of utilities for comparing genomic features. Bioinformatics 2010, 26:841-842

40. Gene Expression Omnibus: http://www.ncbi.n/m.nih.gov/geo/.

41. Mobini R, Andersson BA, Erjefalt J, Hahn-Zoric M, Langston MA, Perkins AD, Cardell LO, Benson M: A module-based analytical strategy to identify novel disease-associated genes shows an inhibitory role for interleukin 7 Receptor in allergic inflammation. BMC Syst Biol 2009, 3:19.

doi:10.1186/1471-2164-13-645

Cite this article as: Gilfillan et al.: Limitations and possibilities of low cell number ChIP-seq. BMC Genomics 2012 13:645.

\section{Submit your next manuscript to BioMed Central and take full advantage of:}

- Convenient online submission

- Thorough peer review

- No space constraints or color figure charges

- Immediate publication on acceptance

- Inclusion in PubMed, CAS, Scopus and Google Scholar

- Research which is freely available for redistribution

Submit your manuscript at www.biomedcentral.com/submit
C Biomed Central 